\title{
Ageism and older people's health and well-being during the Covid-19-pandemic: the moderating role of subjective aging
}

\author{
Anna E. Kornadt ${ }^{1}$ (D) Isabelle Albert $^{1} \cdot$ Martine Hoffmann $^{2} \cdot$ Elke Murdock $^{1} \cdot$ Josepha Nell $^{1}$
}

Accepted: 16 April 2021 / Published online: 30 April 2021

(c) The Author(s) 2021

\begin{abstract}
In the Covid-19 pandemic, being older means being in a special focus: Probabilities for severe infections and mortality rise with increasing age and protective measures for this population group have been increased. This was accompanied by public discourse that portrayed older adults stereotypically as vulnerable and frail but also highlighted the hardships younger people have to endure to protect them. Given the possibly detrimental effects of ageism on individuals and societies, we were interested in older adults' perception of ageism in the Corona-crisis and its relation to their health and well-being. Furthermore, we were interested in subjective aging variables as moderators in the ageism-health relationship. In June 2020, $N=611$ independently living people aged $60+$ from the Grand Duchy of Luxembourg were recruited via a survey research institute and interviewed online or by phone. They reported on perceived ageism in different contexts, their life satisfaction, subjective health, subjective age and self-perceptions of aging. Depending on context, ageism was perceived by around $20 \%$ of participants, and overall negatively related to subjective health and life satisfaction after the onset of the pandemic. Moderated hierarchical regressions showed that a younger subjective age buffered the negative effect of ageism on subjective health, while perceiving aging as social loss increased its effect on life satisfaction. We discuss the importance of addressing and reducing ageism (not only) in times of crisis and the consequences for individuals and societies.
\end{abstract}

Keywords Ageism $\cdot$ Subjective aging $\cdot$ Covid-19 $\cdot$ Well-being $\cdot$ Subjective health $\cdot$ Life satisfaction

\section{Introduction}

The Covid-19 pandemic proves to be challenging for people and societies in various forms. One demographic group that had to deal with particular hardships were older people. Right from the beginning of the pandemic, it was communicated that people over the age of 60 had a higher risk of severe progression of the disease and also increased rates of mortality if contracting the virus (e.g., Onder et al. 2020). This group-based risk assessment (which, for instance, neglects the role of underlying health conditions,

Responsible Editor: Marja J.Aartsen

Anna E. Kornadt

anna.kornadt@uni.lu

1 Department of Behavioural and Cognitive Sciences, University of Luxembourg, 11, Porte des Sciences,

4366 Esch-sur-Alzette, Luxembourg

2 RBS-Center fir Altersfroen, 5955 Itzig, Luxembourg e.g. Montero-Odasso et al. 2020) had pronounced consequences for those included in the risk group: They were particularly advised to strictly follow protocols of protective measures, such as sheltering-in-place and physical distancing (including ceasing physical contact with children and grandchildren), with all the accompanying consequences for their daily lives and well-being (e.g., Krendl and Perry 2020; van Tilburg et al. 2020).

As a positive response to this situation, intergenerational solidarity and support in neighborhoods and institutions emerged, offering help with chores, for instance (e.g., Monahan et al. 2020). On the other hand, public discourse turned towards a narrative of grouping all people over the age of 60 together, into a group requiring shielding and mostly well-meant advice was offered on how this group should behave in the crisis. At the same time, advice was given to other demographic groups on how they should behave in order to protect older people, and-related to this—defend scarce medical and economic resources. This was accompanied by public display of pity and patronizing, but also 
anger directed towards older people. Taken together, these instances showcase pronounced ageism (Ayalon et al. 2020; Lichtenstein 2020; Morrow-Howell and Gonzalez 2020), culminating in reports that in some of the most strongly affected countries and regions, older people did not receive intensive care or ventilation therapy when resources were scarce and doctors had to perform triage decisions under pressure (Cesari and Proietti 2020).

Irrespective of such extreme examples, the perception and experience of ageism can potentially have negative consequences for older adults' health and well-being (e.g., Chang et al. 2020; Monahan et al. 2020), a relationship that might even be exacerbated in times of crisis. Thus, we were interested in the perception of ageism by people over the age of 60 and how it was related to their self-rated health and life satisfaction during the first wave of the Covid-crisis in Luxembourg. Since the consequences of perceived ageism might differ according to people's subjective aging experiences and perceptions of aging, a second question of our study was in how far self-perceptions of aging and subjective age moderated the ageism-outcome relationship.

\section{Ageism and its consequences for individuals and societies}

Ageism, which can be defined as "stereotypes, prejudice, or discrimination against (but also in favor of) people because of their chronological age" (Ayalon and Tesch-Römer 2017, p. 1) can take various forms, and be displayed at different levels, within individuals, organizations, and cultures. At the heart of it is that people are perceived and treated in a certain way because they (are perceived to) belong to a certain age group, irrespective of their actual personal and individual characteristics. While ageism can also take benevolent forms (e.g., Chasteen et al. 2017), it is mostly rooted in the fact that older age is seen as a state of deterioration, loss of functioning and even senescence (Ayalon and Tesch-Römer 2018). Studies show that societally prevalent ageism comes with massive costs for health-care systems and economies (e.g. for the US, Levy et al. 2020). At the individual level, Chang and colleagues (2020) demonstrated in a recent meta-analysis including 422 studies from 45 countries, that ageism transported by institutions, cultural practices, individual perceptions and behaviors negatively impacted all investigated health outcomes (e.g. mortality, hospitalization, quality of life, health behaviors).

Importantly, not only objective instances of ageism are consequential, but the mere perception of being disadvantaged and treated unfairly due to ones' age can also be detrimental (Bratt et al. 2018; Vauclair et al. 2015; Vogt Juan 2007). Being confronted with people's negative perceptions of age might not only directly decrease developmental opportunities of older people, but also turn into self-fulfilling prophecies by becoming entrenched in people's self, bodies and behaviors as they age (Levy 2009; Kornadt et al. 2020). So irrespective of the pandemic context, perceived ageism represents an important risk factor for health and well-being in later life. In a study with data from the European Social Survey, Vauclair and colleagues (2015) showed that perceived ageism predicted self-rated health above and beyond other variables, such as social capital (cf. Armeta et al. 2017). Relatedly, people's life satisfaction is affected by perceived ageism (Garstka et al. 2004). Given that self-rated health and life satisfaction are frequently used and easily assessable variables with high importance for development in later life, we use both indicators as outcome variables in the present study.

\section{Manifestations of ageism during the pandemic}

Only 10 months into the pandemic as of the time of writing, there has already been an abundance of papers in scientific journals and other outlets detailing the Covid-related increase of ageism. In one of the first review articles on the topic, Ayalon and colleagues (2020) impressively detailed instances of ageism that were found in newspapers all over the world. Those ranged from naming the virus "Boomer Remover" to suggesting that older adults should sacrifice themselves for the younger generations and the economy (see also examples in Meisner 2020; Wahl and Ehni 2020). Messages disseminated followed two trajectories: The "vulnerability narrative" focused on the group of older people as a uniform, undifferentiated group of fragile, vulnerable people, who should be protected at any cost. The "burden narrative", on the other hand, focused on the hardships that younger people had to endure because they had to protect older people (Cohn-Schwartz and Ayalon 2020). More anecdotal evidence reported in the reviews and appeals was supported by systematic analyses. In an analysis of newspapers and other media outlets in Australia, the UK and the US, Liechtenstein (2020) showed that in all three countries, ageist and age-centric debates were prevalent, especially at the onset of the pandemic. (Xiang et al. 2020) investigated more than 80.000 tweets with a machine learning approach and found that on average $20 \%$ of these tweets had ageist content, devaluing older adults' lives or ridiculing them. It has to be noted, however, that the most extreme of these posts (e.g. the suggestions by a Texan politician that older adults should sacrifice their lives if it will mitigate the pandemic's economic damage to younger people) were also met with broad opposition and critique (Barrett et al. 2020).

Yet besides those narratives and articles describing the phenomenon, few papers have empirically dealt with the perception of ageism by older people themselves and the consequences as well as moderators of this relationship. Cohn-Schwartz and Ayalon (2020) for example asked Israeli 
adults aged 50+ whether they perceive ageism to be prevalent in Israeli society during the crisis, and found this was the case especially for the idea that older people are particularly vulnerable. About one quarter of their participants also reported that they experienced ageism in the health care system during the pandemic. In another study from Israel, Bergman et al. (2020) found that participants with higher self-reported ageism had higher anxiety levels during the pandemic. However, both studies did not directly look at the relationship of people's own experiences of perceived ageism with health outcomes and what might moderate this relationship.

\section{Perceptions of subjective aging as moderators of the ageism-health relationship}

Besides ageism directed at people by others or towards others, perceptions of subjective aging play a central role for personal development, health and well-being across the lifespan (Kornadt et al. 2020). Subjective aging is defined as "individuals' experiences with their own aging process and the state of being old" and it includes self-perceptions of aging, as well as subjective age (Wurm et al. 2017, p. 28). Whereas self-perceptions of aging include ideas about one's own aging process, subjective age is defined as the age one feels like relative to one's chronological age (Kotter-Gruhn et al. 2016). Both variables have been shown to impact health, well-being and mortality of older people in a large number of studies with a variety of different operationalizations, samples and outcomes (for a meta-analysis see Westerhof et al. 2014). Their relevance in the Covid-19 pandemic was also demonstrated in one of the first empirical studies on well-being during the crisis. Losada-Baltar and colleagues (2020) found in their large sample of Spaniards aged 18-88 that people with more negative perceptions of their own aging (as measured with the widely used Atitudes Toward Own Aging scale by Lawton), perceived higher loneliness and more psychological distress in the first weeks of lockdown.

Besides the direct effect of subjective aging on health outcomes, those perceptions can also function as moderator in the relationship between predictors and health outcomes in later life. In the context of post-traumatic stress disease (PTSD) for example, people who felt comparatively older had a stronger negative relationship between PTSD symptoms and for instance physical functioning (Shrira et al. 2018). Feeling older also increased the relationship between depressive symptoms and physical morbidity in a longitudinal study (Segel-Karpas et al. 2017). Relatedly, self-perceptions of aging as physical decline moderated the use of self-regulatory strategies in case of a serious health event, with those participants that perceived aging more as physical decline engaging in less strategies
(Wurm et al. 2013). (Han 2018) also found that declining functional health led to more depressive symptoms if people had more negative self-perceptions of aging. With regard to the relationship between ageism, health and wellbeing, the assumption that self-perceptions of aging and subjective age might act as moderators has actually been demonstrated in experimental studies (e.g., Weiss et al. 2013): For those people who feel they belong to the older age group (as indicated by higher subjective age) and also focus on the negative characteristics of aging (as indicated by higher perceptions of aging as physical decline and social loss), experiences of ageism might possess more self-relevance and threat potential and thus also affect them more strongly (Levy 2009).

It has to be noted that views on aging are a domain-specific construct (for an overview, see Kornadt et al. 2020). Besides, views on aging show stronger relationships to outcomes that match their respective content, this has been termed the stereotype matching effect (Levy and LeifheitLimson 2009). Domain-specificity thus needs to be taken into account when linking different views on aging to various outcome variables.

\section{Aims and hypotheses of the current study}

Given the notable increase and prevalence of ageism during the Covid-19 pandemic and the detrimental consequences that ageism might have on the health and wellbeing of older adults themselves, but also on a society, we were interested in the perception of ageism during the first wave of the Covid-crisis in Luxembourg. We asked people aged 60 and older if they had been treated unfairly due to their age in different contexts during the pandemic. We expected higher perceptions of ageist treatment to be related to worse subjective health and life satisfaction (H1). Furthermore, we were interested in the moderating role of self-perceptions of aging in different domains as well as subjective age for the relationship between ageism and outcomes. We expected a younger subjective age and self-perceptions of aging as continued growth to have a positive effect on subjective health and life satisfaction (H2a), but also a buffering effect in the relationship between ageism and life satisfaction (H3a). In contrast, an older subjective age and self-perceptions of aging as social loss and physical decline should be negatively related to subjective health and life satisfaction ( $\mathrm{H} 2 \mathrm{~b})$, and also exacerbate the relationship between ageism and the outcome variables (H3b). According to theories of stereotype matching (Levy and Leifheit-Limson 2009), we expected a more pronounced relationship between self-perceptions of aging as physical decline and subjective health, and self-perceptions of aging as social loss and life satisfaction 


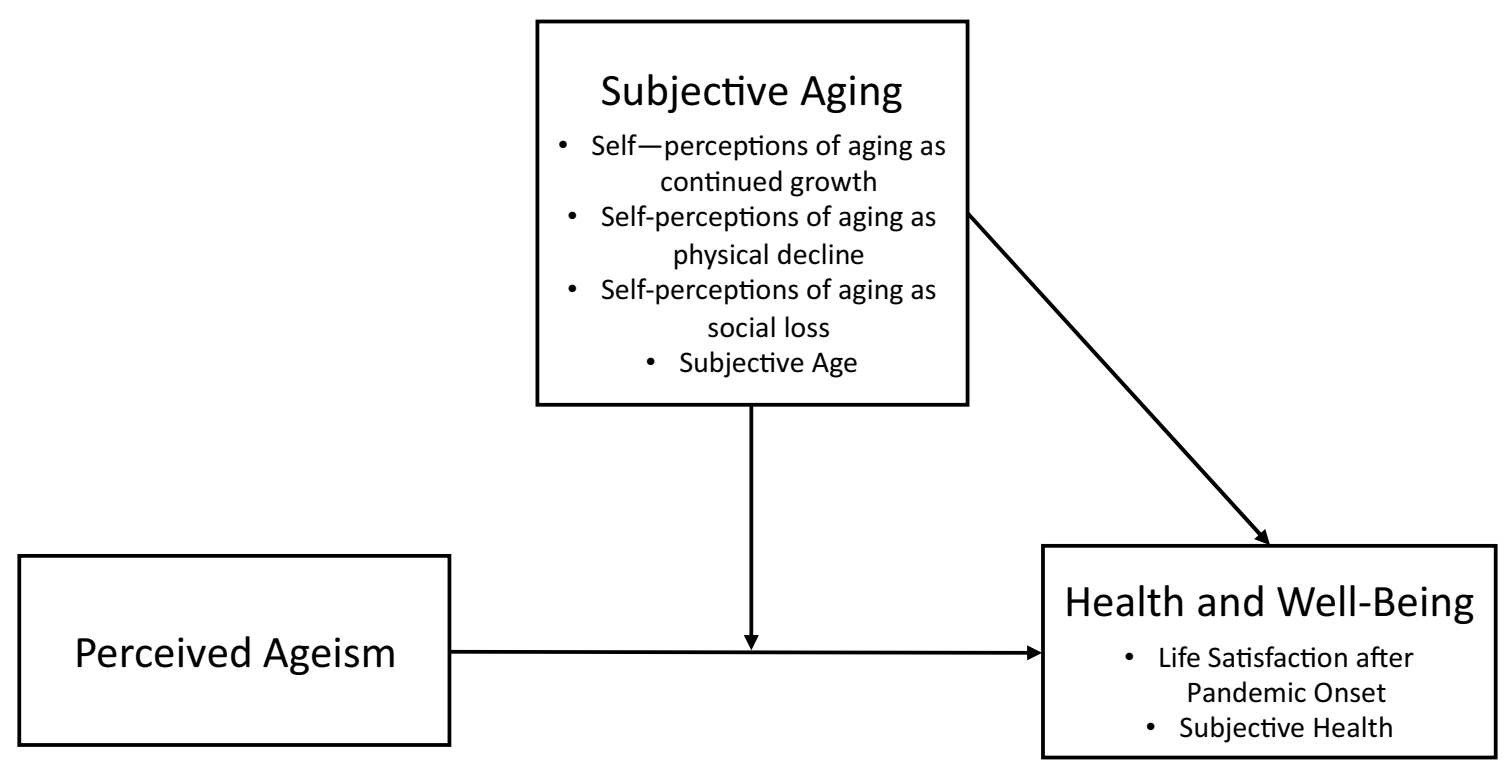

Fig. 1 Schematic depiction of investigated relationships between perceived ageism and health and well-being outcomes, moderated by subjective aging variables

(H4). Investigated relationships between all variables are schematically presented in Fig. 1.

\section{Method}

\section{Sample and procedure}

Our sample consisted of $N=611$ community-dwelling participants aged $60-98\left(M_{\text {age }}=69.92, S D=6.97\right) .49 .6 \%$ of the sample were female and $29.5 \%$ reported at least some tertiary education. They were recruited in June 2020 by a survey research institute (TNS ILRES) in Luxembourg. The survey was carried out either by phone, for which participants were recruited via random digit dialing ( $n=240$, response rate $27 \%$ ), or online, recruited from a large database of persons who agreed to be contacted for online surveys $(n=371$, response rate $40 \%$ ). Participants who completed the survey on the phone were older $[\mathrm{t}(606)=6.94, p<0.001]$, reported less perceived ageism $[\mathrm{t}(607)=-4.08, p<0.001]$, lower subjective health $\mathrm{t}(607)=-3.25, p=0.001]$, and lower life satisfaction before Corona [ $\mathrm{t}(606)=-2.04, p=0.04]$. There were no significant differences for the subjective aging variables. The questionnaire assessed sociodemographic information, the perception of the Covid-crisis in Luxembourg in general, their personal situation in the crisis, perceived ageism, subjective aging, and a number of other risk and resilience factors. The study was approved by the Ethics Review Panel of the University of Luxembourg (ERP 20-042-C CRISIS).

\section{Measures}

\section{Perceived ageism}

We assessed perceived ageism by asking people "During the Covid-19 pandemic, have you felt that you were treated unfairly due to your age in the following domains": 1) media coverage, 2) health care, 3 ) activities of daily life (e.g. shopping), and 4) within my social network (friends, family). ${ }^{1}$ These contexts were selected because of their relevance in previous research on ageism (e.g., Chasteen et al. 2017) and due to their relevance in the context of the pandemic. Participants had to indicate whether they felt unfairly treated very strongly, strongly, somewhat or not at all. We collapsed these items into one index of perceived ageism (Cronbach's $\alpha=0.75$ ) and recoded the items so that higher values represent more perceived ageism.

\section{Self-perceptions of aging}

Self-perceptions of aging were assessed with the established AgeCog scales (Steverink et al. 2001) which assess people's perception of their own aging ("Aging means to me...") in the domains of physical decline (3 items, e.g."...that my

\footnotetext{
1 We also assessed perceived ageism in the work context. However, due to the fact that $90 \%$ of our participants were already retired, this domain was not relevant for most and thus, $n=372$ participants (61\%) did not report on this item. Therefore, we excluded this item from the index. Results did not differ for both versions of the index.
} 
health is declining", $\alpha=0.80$ ), continued growth (three items, e.g., " "..that I continue to make plans", $\alpha=0.72$ ) and social loss (four items, e.g. "... that I feel lonely more often", $\alpha=0.67)$. Participants had to rate the items on a four-point scale from completely applies to does not apply at all. Values were also recoded so that higher values represent higher endorsement of the respective scale.

\section{Subjective age}

Subjective age was assessed with one item asking people "Aside from your actual age: How old do you feel, in years?". This number was subtracted from people's chronological age, with more negative values indicating feeling younger than one's chronological age. According to conventions, values of three standard deviations above and below the mean were removed (people who felt more than 38 years younger or more than 18 years older than their chronological age, $1.3 \%$ of cases).

\section{Subjective health}

Participants had to indicate their current state of health on a five-point scale ranging from very good to very bad. Again, values were recoded so that higher values indicated better subjective health.

\section{Life satisfaction before and since the onset of the pandemic}

Participants were asked to rate how satisfied they were with their life as a whole 1) before the spread of the CoronaVirus, and 2) since the spread of the Corona-Virus, respectively, on a four-point scale from very satisfied to very unsatisfied. Values were recoded so that higher values indicate more satisfaction.

\section{Analyses}

We first computed descriptive statistics and correlations for the variables of interest to address means and bivariate relationships. To address whether perceived ageism was related to subjective health and life satisfaction and whether selfperceptions of aging and subjective age moderated the relationship between ageism and the outcome variables, we ran hierarchical moderated regression analyses with IBM SPSS 26 for both outcome variables separately. In a first step, age (and in the case of life satisfaction, satisfaction before the onset of the pandemic) were regressed on subjective health and life satisfaction after the onset of the pandemic, respectively. In a second step, perceived ageism was added as a predictor, in a third step the four subjective aging variables, and in a fourth step the interaction terms between ageism and the subjective aging variables. All predictor variables were standardized prior to analyses and the computation of interaction terms. When interactions were significant, we repeated the analyses for this predictor alone with the process macro (Hayes 2017) in order to probe the interaction. Due to listwise deletion of missing values, analysis $\mathrm{N}$ for the moderated regressions was $N=527$ for the analysis with life satisfaction as outcome and $N=528$ for the analyses with subjective health as outcome.

\section{Results}

\section{Descriptive statistics and bivariate relations}

Means and standard deviations as well as bivariate correlations between all study variables are presented in Table 1 . On a mean level, participants reported relatively little ageism $(M=1.27, S D=0.50)$, mostly in the media $(M=1.35$, $S D=0.76)$, medical treatment $(M=1.32, S D=0.72)$, and activities of daily life $(M=1.26, S D=0.63)$, least in their social networks $(M=1.15, S D=0.49)$. The overall perception of ageism was unrelated to age $(r=-0.03, p=0.55)$. In absolute numbers, however, a considerable number of people reported to have been at least somewhat treated unfairly due to their age during the pandemic (media: 20,7\%; health care: 19\%; daily activities: $17,5 \%$; family: $10,8 \%$ ).

Participants reported to be satisfied with their life, however, compared to their life satisfaction before the onset of the pandemic $(M=3.26, S D=0.50)$, participants reported less life satisfaction after the onset of the pan$\operatorname{demic}(M=3.05, S D=0.54), F(1,604)=105.70, p<0.001$, $\eta_{\mathrm{p}}{ }^{2}=0.15$. Subjective health was rated overall to be good $(M=4.03, S D=0.73)$. With regard to subjective aging, participants felt around 10 years younger than their actual age, and perceived their own aging to be rather accompanied by continued growth and physical decline and only somewhat by social loss.

Higher perceived ageism was related to lower life satisfaction before and after the onset of the pandemic, and also to worse subjective health. In addition, more perceived ageism was related to perceiving aging as being accompanied by more social loss and physical decline but was unrelated to the perception of continued growth and participants' subjective age. The subjective aging variables were also related to subjective health and life satisfaction, with more continued growth, and lower subjective age as well as less physical decline and social loss accompanied with better subjective health and life satisfaction before and after Corona. Subjective age, however, was unrelated to life satisfaction before Corona. 


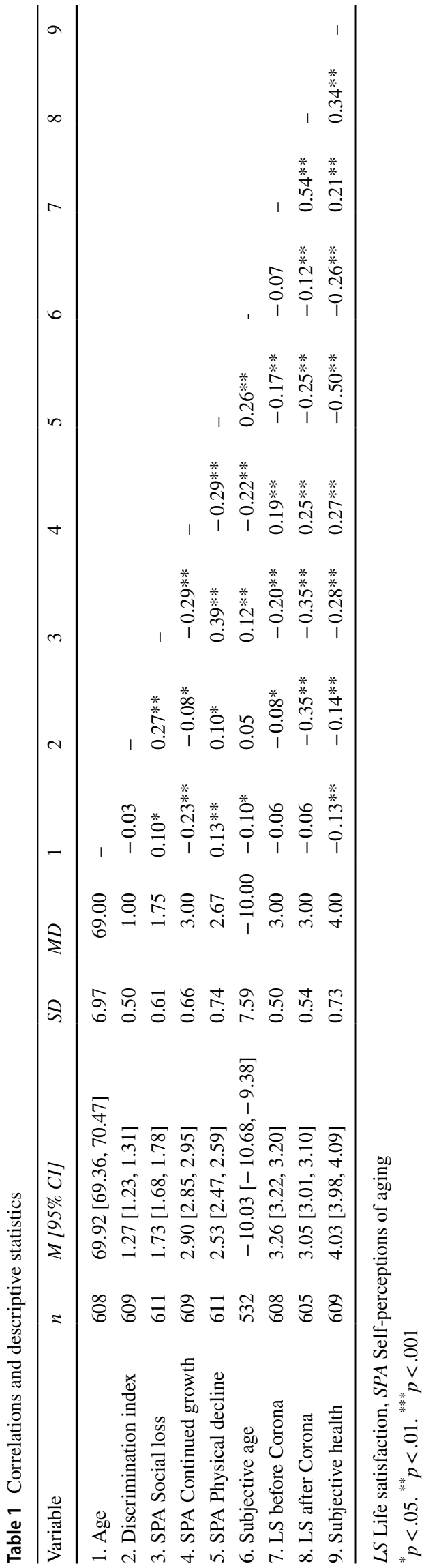

\section{Moderated hierarchical linear regressions}

\section{Life satisfaction}

Results of the analyses with life satisfaction after the onset of the Corona pandemic as outcome variable are displayed in Table 2. Controlling for age and life satisfaction before Corona, ${ }^{2}$ perceived ageism was still negatively related to life satisfaction after the onset of the pandemic $(\beta=-0.30$, $p<0.001)$. Including the subjective aging variables significantly increased the amount of explained variance. In particular, perceiving one's own aging as accompanied by more social loss was negatively related to life satisfaction ( $\beta=-0.16, p<0.001)$, whereas the perception of continued growth was positively related to life satisfaction $(\beta=0.10$, $p=0.006$ ). Entering the interactions between perceived ageism and the subjective aging variables yielded more explained variance and a significant interaction between social loss and ageism: For those who perceived their aging as accompanied by more social loss, the experience of ageism during the crisis had an especially negative effect on life satisfaction (Fig. 2).

\section{Subjective health}

Results for subjective health as the outcome variable are displayed in Table 3. Controlling for age, perceived ageism was negatively related to subjective health $(\beta=-0.12$, $p=0.004)$. The subjective aging variables incrementally explained variance in subjective health, perceiving aging accompanied by physical decline $(\beta=-0.40, p<0.001)$ and an older subjective age $(\beta=-0.14, p<0.001)$ were related to decreased subjective health. Again, including the interaction terms increased explained variance. Those who perceived more ageism had worse subjective health especially when they also had higher subjective age (Fig. 3). In addition, including the interaction terms rendered the main effect of continued growth significant $(\beta=0.08, p=0.048)$, which was not significant in the step including the main effects only.

\section{Discussion}

In light of the numerous reports of ageism during the Covidcrisis, our study provides empirical evidence from the perspective of older adults themselves. We focused on Luxembourgish older adults' perception of having been treated

\footnotetext{
${ }^{2}$ Results for both regression analyses did not change when including gender, education, living situation (alone vs. with others) and survey mode as additional covariates.
} 
Table 2 Hierarchical regression results with life satisfaction since the beginning of the crisis as outcome

\begin{tabular}{|c|c|c|c|c|c|c|c|}
\hline Variable & $B$ & $S E B$ & $\beta$ & $p$ & $R^{2}$ & $p$ & $\Delta R^{2}$ \\
\hline Step 1 & & & & & 0.27 & $<0.001$ & $0.27 *$ \\
\hline Constant & 1.54 & 0.25 & & $<0.001$ & & & \\
\hline LS before Corona & 0.56 & 0.04 & 0.52 & $<0.001$ & & & \\
\hline Age & -0.00 & 0.00 & -0.05 & 0.149 & & & \\
\hline Step 2 & & & & & 0.36 & $<0.001$ & $0.09 *$ \\
\hline Constant & 1.63 & 0.23 & & $<0.001$ & & & \\
\hline LS before Corona & 0.54 & 0.04 & 0.50 & $<0.001$ & & & \\
\hline Age & -0.01 & 0.00 & -0.06 & 0.083 & & & \\
\hline Discrimination index & -0.17 & 0.02 & -0.30 & $<0.001$ & & & \\
\hline Step 3 & & & & & 0.41 & $<0.001$ & $0.05^{*}$ \\
\hline Constant & 1.57 & 0.23 & & $<0.001$ & & & \\
\hline LS before Corona & 0.48 & 0.04 & 0.45 & $<0.001$ & & & \\
\hline Age & -0.00 & 0.00 & -0.02 & 0.614 & & & \\
\hline Discrimination index & -0.14 & 0.02 & -0.25 & $<0.001$ & & & \\
\hline SPA social loss & -0.09 & 0.02 & -0.16 & $<0.001$ & & & \\
\hline SPA continued growth & 0.06 & 0.02 & 0.10 & 0.006 & & & \\
\hline SPA physical decline & -0.03 & 0.02 & -0.06 & 0.140 & & & \\
\hline Subjective age & -0.01 & 0.02 & -0.01 & 0.712 & & & \\
\hline Step 4 & & & & & 0.42 & 0.015 & $0.01 *$ \\
\hline Constant & 1.54 & 0.23 & & $<0.001$ & & & \\
\hline LS before Corona & 0.48 & 0.04 & 0.45 & $<0.001$ & & & \\
\hline Age & -0.00 & 0.00 & -0.01 & 0.743 & & & \\
\hline Discrimination index & -0.11 & 0.02 & -0.20 & $<0.001$ & & & \\
\hline SPA Social loss & -0.09 & 0.02 & -0.16 & $<0.001$ & & & \\
\hline SPA Continued growth & 0.06 & 0.02 & 0.10 & 0.007 & & & \\
\hline SPA Physical decline & -0.03 & 0.02 & -0.05 & 0.187 & & & \\
\hline Subjective age & -0.01 & 0.02 & -0.02 & 0.645 & & & \\
\hline Discr x Social loss & -0.07 & 0.02 & -0.13 & 0.005 & & & \\
\hline Discr $x$ Continued growth & 0.01 & 0.02 & 0.00 & 0.667 & & & \\
\hline Discr $x$ Physical decline & 0.00 & 0.02 & 0.01 & 0.869 & & & \\
\hline Discr x Subjective age & -0.01 & 0.02 & -0.02 & 0.474 & & & \\
\hline
\end{tabular}

$L S$ Life satisfaction, SPA Self-perceptions of aging; * $p<.05$ unfairly due to their age during the first months of the pandemic. While levels of perceived ageism were relatively low for all domains assessed, around $20 \%$ of participants reported to have been treated somewhat unfairly due to their age in the media, health care and in their daily activities. This is comparable to data from Israel as reported by CohnSchwartz and Ayalon (2020). Besides, individual differences in perceived ageism mattered for participants' subjective health and reported life satisfaction during the crisis: Those who reported to have experienced more ageism during the crisis also reported lower subjective health and life satisfaction after the onset of the crisis, even when controlled for reported life satisfaction before the onset of the crisis, which supports our first hypothesis. This is in line with previous studies linking perceived ageism to life satisfaction and self-rated health (Garstka et al. 2004; Vauclair et al. 2015). Given that both variables are important indicators of health and well-being (Whitley et al. 2016), this provides further evidence for the impact of perceived ageism on successful aging (Chang et al. 2020).

Albeit correlational, these findings support the numerous researchers and representatives who have warned about the negative effects of ageist narratives and actions during the pandemic (e.g. Ayalon et al. 2020, Meisner 2020; Wahl and Ehni 2020). While it is of course necessary and advisable for societies to protect its most vulnerable and in-need members, especially in times of heightened risk and crisis, simplifications in risk communication, ignoring individual differences within societal groups, and thereby fueling intergenerational conflict might have profound effects on individuals and also on societies as a whole in the long run.

Since negative age-related communication and actions have a larger impact on individuals when they have stronger self-relevance and are met with pre-existing negative 


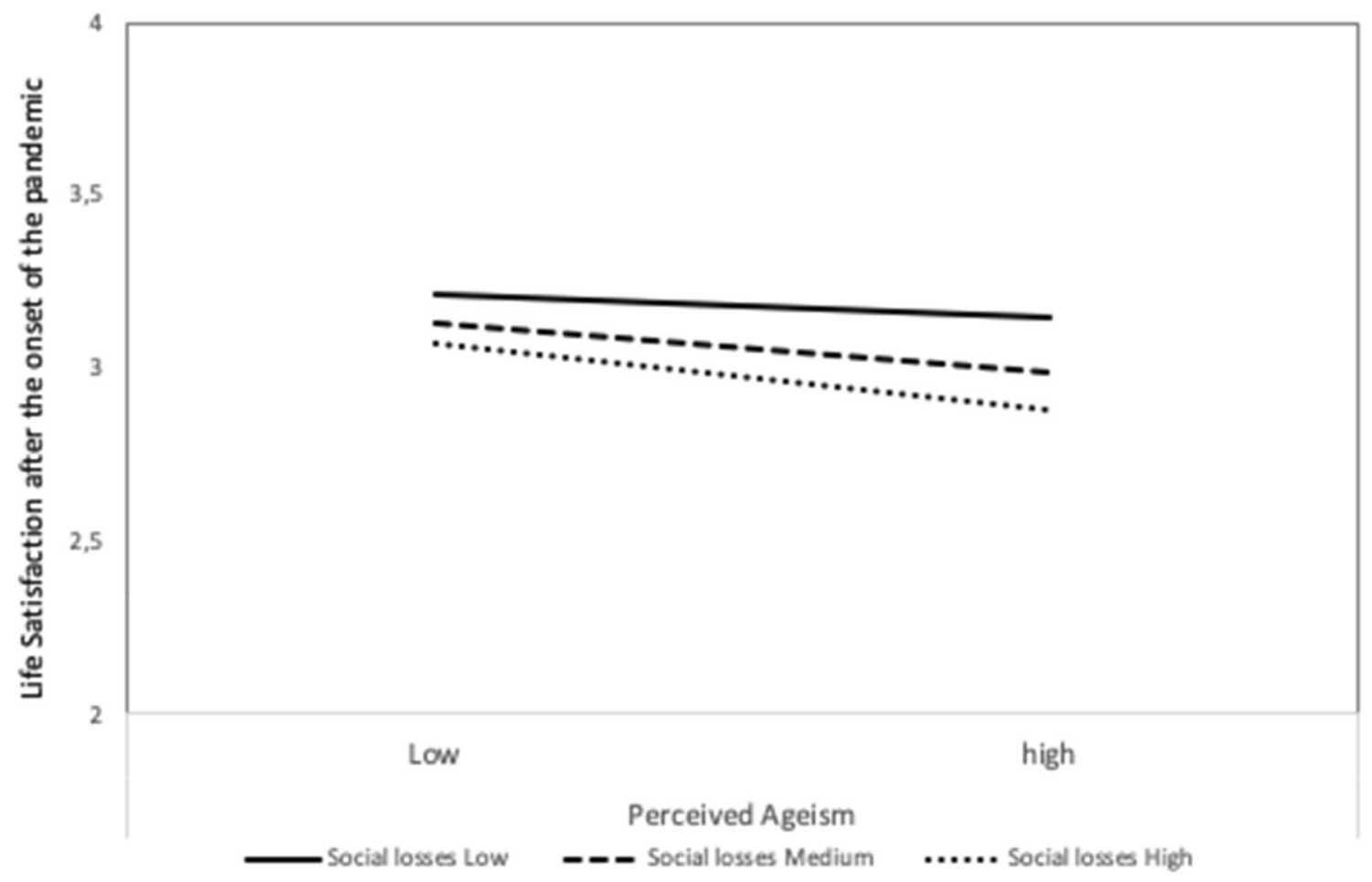

Fig. 2 Effect of perceived ageism on life satisfaction after the onset of the pandemic for different values of self-perceptions of aging as social loss $($ low $=16$ th percentile, medium $=50$ th percentile, high 84 th percentile $)$

perceptions about one's own aging, we investigated the moderating role of self-perceptions of aging and subjective age. Supporting our hypotheses $\mathrm{H} 2 \mathrm{a}$ and $\mathrm{H} 2 \mathrm{~b}$, and in line with previous studies that show the relevance of subjective aging for development especially in the second half of life (e.g., Wurm et al. 2017), we found several main effects for the subjective aging variables on life satisfaction and subjective health. Those who perceived their own aging as accompanied by continued growth had better life satisfaction after the onset of the crisis and feeling younger than one's actual age acted as a resource for better health. Contrary to other studies (Westerhof et al. 2014) it was however not related to life satisfaction. In line with assumptions regarding the domain-specificity of views on aging and the stereotype matching hypothesis (Levy and Leifheit-Limson 2009), selfperceptions of aging as social loss were only related to life satisfaction and self-perceptions of aging were only related to subjective health, i.e., the outcome domains that best matched their respective content. This supports our fourth hypothesis and again highlights the need for a differentiated assessment of views on aging when predicting diverse developmental outcomes (Kornadt et al. 2020).

In line with our hypotheses $\mathrm{H} 3 \mathrm{a}$ and $\mathrm{H} 3 \mathrm{~b}$, we found evidence that views on aging moderated the ageism-outcome relationship and thus can act as risk factors and resources in challenging situations. However, this was only true for subjective age and self-perceptions of aging as social loss, while, contrary to our expectations, self-perceptions of aging as continued growth and physical decline did not act as buffers or amplifiers for the relation between ageism and outcomes. Those who felt younger were less affected in their subjective health when they perceived ageism during the crisis. It seems that distancing oneself from ones' age group and thus in many cases from the proclaimed risk population seems to be beneficial for health-related outcomes when there is a perceived threat to this group, at least in the short run. This is in line with previous experimental research (Weiss et al 2013). However, the question is whether there also might be negative consequences of such distancing on other indicators, which might compromise or facilitate the compliance with safety measures and risk perception (e.g., Bruine de Bruin and Bennett 2020). Paradoxically, excluding oneself from the risk group might actually lead to a heightened risk of actual infection; this possibility and the implications for older adults need to be addressed in future studies with different timelines.

Stronger endorsement of self-perceptions of aging as social loss also increased the negative relation of ageism and life satisfaction. Having a strong belief that one's own aging is or will be accompanied by increases in loneliness, boredom and uselessness, as well as decreases in respect seems to increase older adults' susceptibility to negative messages 
Table 3 Hierarchical regression results with subjective health as outcome variable

\begin{tabular}{|c|c|c|c|c|c|c|c|}
\hline Variable & $B$ & $S E B$ & $\beta$ & $p$ & $R^{2}$ & $p$ & $\Delta R^{2}$ \\
\hline Step 1 & & & & & 0.03 & $<0.001$ & $0.03^{*}$ \\
\hline Constant & 5.19 & 0.31 & & $<0.001$ & & & \\
\hline Age & -0.02 & 0.00 & -0.16 & $<0.001$ & & & \\
\hline Step2 & & & & & 0.04 & 0.003 & $0.02 *$ \\
\hline Constant & 5.21 & 0.31 & & $<0.001$ & & & \\
\hline Age & -0.02 & 0.00 & -0.16 & $<0.001$ & & & \\
\hline Discrimination index & -0.09 & 0.03 & -0.12 & 0.004 & & & \\
\hline Step 3 & & & & & 0.30 & $<0.001$ & $0.26^{*}$ \\
\hline Constant & 4.76 & 0.28 & & $<0.001$ & & & \\
\hline Age & -0.01 & 0.00 & -0.10 & 0.011 & & & \\
\hline Discrimination index & -0.04 & 0.03 & -0.06 & 0.147 & & & \\
\hline SPA Social loss & -0.04 & 0.03 & -0.06 & 0.135 & & & \\
\hline SPA Continued growth & 0.06 & 0.03 & 0.07 & 0.068 & & & \\
\hline SPA Physical decline & -0.28 & 0.03 & -0.40 & $<0.001$ & & & \\
\hline Subjective age & -0.10 & 0.03 & -0.14 & 0.001 & & & \\
\hline Step 4 & & & & & 0.33 & $<0.001$ & $0.04 *$ \\
\hline Constant & 4.72 & 0.27 & & $<0.001$ & & & \\
\hline Age & -0.01 & 0.00 & -0.09 & 0.016 & & & \\
\hline Discrimination index & -0.02 & 0.03 & -0.04 & 0.373 & & & \\
\hline SPA Social loss & -0.05 & 0.03 & -0.07 & 0.097 & & & \\
\hline SPA Continued growth & 0.06 & 0.03 & 0.08 & 0.048 & & & \\
\hline SPA Physical decline & -0.29 & 0.03 & -0.41 & $<0.001$ & & & \\
\hline Subjective age & -0.09 & 0.03 & -0.13 & 0.001 & & & \\
\hline Discr x Social loss & 0.02 & 0.03 & 0.03 & 0.556 & & & \\
\hline Discr $x$ Continued growth & 0.04 & 0.03 & 0.04 & 0.301 & & & \\
\hline Discr x Physical decline & 0.04 & 0.03 & 0.05 & 0.309 & & & \\
\hline Discr x Subjective age & -0.14 & 0.03 & -0.19 & $<0.001$ & & & \\
\hline
\end{tabular}

SPA Self-perceptions of aging; $* p<.05$ about their age group. This might be especially pronounced in the current pandemic, due to the focus on distance as a key factor in preventing the spread of the disease. Older adults with negative beliefs about aging regarding social loss might perceive the current situation and the negative societal atmosphere toward older adults as the starting point for inevitable, irreversible age-related decline. This in turn might lead to those perceptions becoming self-fulfilling prophecies (Levy 2009). Again, the long-term impact and the possibility of a rebound in life satisfaction after the preventive measures have been lifted need to be addressed in future studies.

\section{Limitations and implications for future research}

While our study has several strengths, such as the large sample and the differential assessment of perceived ageism and subjective aging variables, some limitations have to be noted that at the same time point to opportunities for future research. First of all, our study is correlational in nature, thus, inferences about causality or directionality cannot be drawn. Even though the directional relationship between ageism and developmental outcomes in later life has been shown by previous longitudinal and experimental studies, older people with lower life satisfaction and subjective health might more readily perceive instances of ageism. Since the relationships might also differ according to the timeline applied (e.g., short-term, vs. weeks, vs. months), longitudinal studies with different time intervals are needed to pursue the long term consequences of ageism triggered by the Covid-crisis for the future (personal) development of older people.

Since our study was not prospective in nature, we did not have a value for life satisfaction at our disposal that was actually assessed before the onset of the pandemic, but had to rely on retrospective ratings, which might have been biased. However, we still consider them to be a good approximation, since they capture the discrepancies between the time before and after the onset of the pandemic as they were perceived by our participants at the time of the assessment. Relatedly, we did not have any other retrospective measure, for example for subjective health to control for, and also no other indicators of health problems, which might have impacted our 


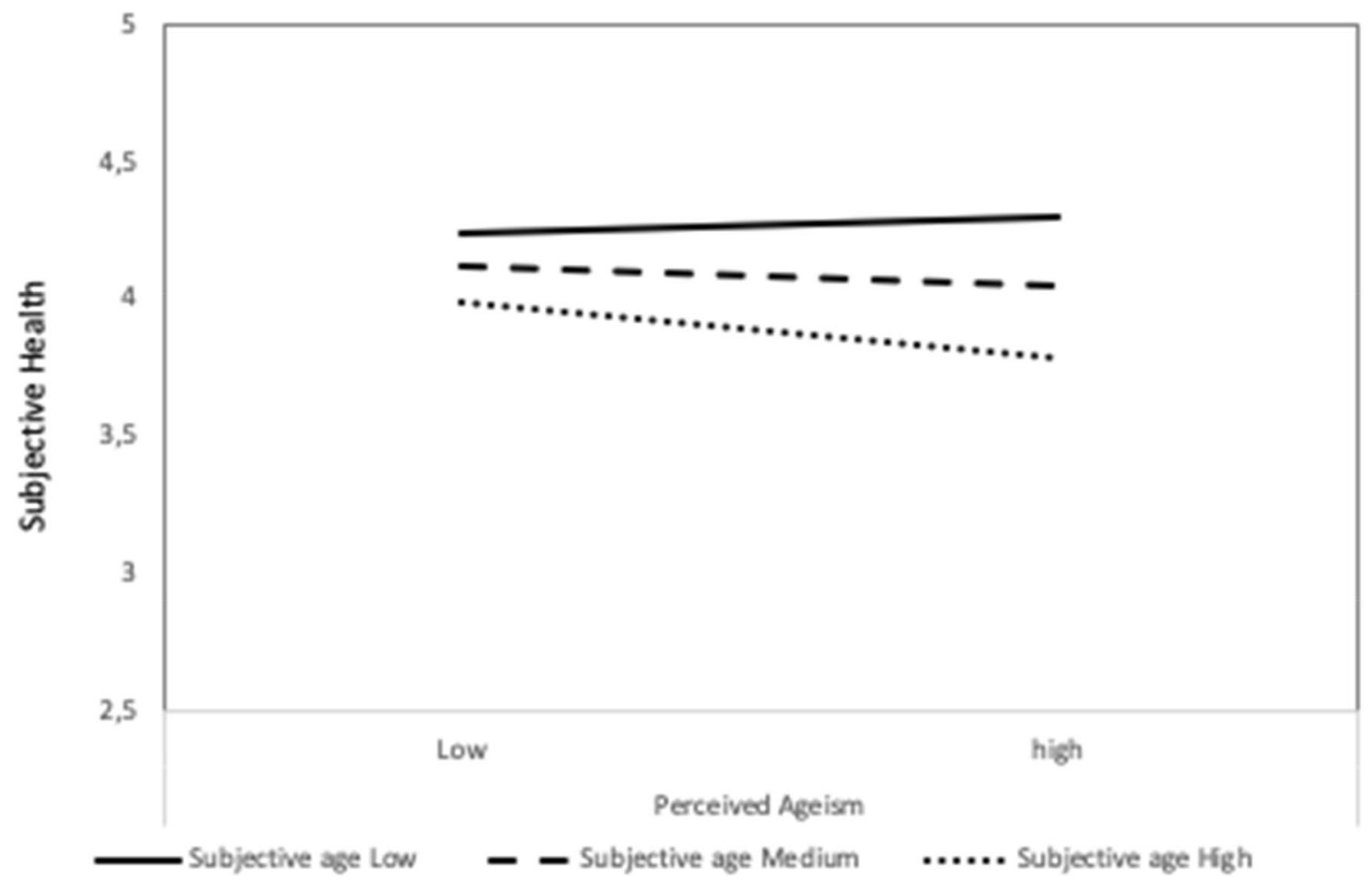

Fig. 3 Effect of perceived ageism on subjective health for different values of subjective age (low $=16$ th percentile, medium $=50$ th percentile, high 84th percentile)

findings. Besides, the perception of ageism is also dependent on factors we did not control for: For instance, participants with family members are at higher risk of perceiving ageism in the family compared to those without family; people who use health care more frequently may be more susceptible to ageism in this domain. ${ }^{3}$ These structural factors should be addressed in future research.

Even though we were careful to assess perceived ageism in a differentiated way and absolute numbers were similar to studies from other countries (Cohn-Schwartz \& Ayalon, 2020), we asked people about "being treated unfairly" due to their age. Even though similarly worded questions have been used in other studies (e.g., Bratt et al 2018), this might put the focus on malevolent instances of ageism and not bring to mind benevolent ageism (such as the well-meant take-over of chores, regardless of people's wishes), which might still have negative effects, perhaps more on an implicit and long-term level, for example by reducing autonomy and self-worth (Monahan et al. 2020). These effects also need closer inspection in future studies. Besides, even though our measure assessed perceived ageism in different domains, we decided to collapse them

\footnotetext{
$\overline{3}$ We thank an anonymous reviewer for pointing out this important point.
}

into one index for psychometric reasons and parsimony, at the expense of a differentiated view of ageism in different domains and on different levels (individual, societal). This is an important distinction and needs to be addressed in more detail in future studies (Ayalon and Tesch-Römer 2017, 2018).

Our sample consisted of community-dwelling, independently living, mostly middle-class older adults who reported to have good health and being rather satisfied with their lives. It will also be meaningful to address the questions of ageism in contexts where the pandemic and the protective measures taken might have an even stronger impact on older people's health and well-being. This might be true for older people living in unfavorable (e.g., in poverty, poor health, violent contexts) and also in institutionalized contexts. Since much press coverage was also centered at older adults in homes or hospitals, they might bear the double burden of increased risks from the disease and ageism even stronger.

\section{Conclusion}

To conclude, our study provides one of the first empirical investigations of perceived ageism and views on aging during the Covid-crisis and its relationship to the health and well-being of older people. The perception of ageism might 
affect this demographic group profoundly, especially in times of uncertainty and hardship. Propositions to mitigate ageism and its consequences in the crisis have been made (e.g., Wahl and Ehni 2020) and need to find application in management of risk communication, intergenerational relationships and support as well as public discourse. For instance, the communication enhancement model of aging proposed by (Ryan and colleagues 1995) might be used to develop non-discriminatory risk communication and coping strategies: Communicating in a non-discriminatory manner the necessity of preventive measures, and at the same time recognizing the diversity of older people, their resources, experiences, competences and needs, as well as their role as active and valuable members of society. This approach should result in a more productive and benevolent societal discourse and more favorable outcomes for individuals and (aging) societies alike, especially but not only in times of crisis.

Authors' contributions Isabelle Albert wrote the CRISIS study proposal, and all authors contributed to the current paper's conception, design and the preparation of the data collection, which was performed by a survey institute (TNS ILRES, Luxembourg). Data preparation and analyses were performed by Anna E. Kornadt. The first draft of the manuscript was written by Anna E. Kornadt with feedback by Isabelle Albert and all authors commented on previous versions of the manuscript. All authors read and approved the final manuscript.

Funding The project CRISIS is funded by a grant of the Luxembourg National Research Fund to Isabelle Albert (Ref.: 14705672-CRISIS).

Availability of data and material All data and materials can be obtained from the authors upon request.

Code availability The SPSS syntax used for all analyses can be obtained from the first author upon request.

\section{Declarations}

Conflicts of interest The authors report no conflicts of interest.

Ethics Approval The CRISIS study was approved by the Ethics Review Panel of the University of Luxembourg (Ref.: ERP 20-042-C CRISIS).

Open Access This article is licensed under a Creative Commons Attribution 4.0 International License, which permits use, sharing, adaptation, distribution and reproduction in any medium or format, as long as you give appropriate credit to the original author(s) and the source, provide a link to the Creative Commons licence, and indicate if changes were made. The images or other third party material in this article are included in the article's Creative Commons licence, unless indicated otherwise in a credit line to the material. If material is not included in the article's Creative Commons licence and your intended use is not permitted by statutory regulation or exceeds the permitted use, you will need to obtain permission directly from the copyright holder. To view a copy of this licence, visit http://creativecommons. org/licenses/by/4.0/.

\section{References}

Ayalon L, Tesch-Romer C (2017) Taking a closer look at ageism: selfand other-directed ageist attitudes and discrimination. Eur J Ageing 14:1-4. https://doi.org/10.1007/s10433-016-0409-9

Ayalon L, Tesch-Römer C (2018) contemporary perspectives on ageism. Springer, Cham

Ayalon L et al (2020) Aging in times of the COVID-19 pandemic: avoiding ageism and fostering intergenerational solidarity. J Gerontol B Psychol Sci Soc Sci. https://doi.org/10.1093/geronb/gbaa0 51

Barrett AE, Michael C, Padavic I (2020) Calculated ageism: generational sacrifice as a response to the COVID-19 pandemic. J Gerontol B Psychol Sci Soc Sci. https://doi.org/10.1093/geronb/gbaa132

Bergman YS, Cohen-Fridel S, Shrira A, Bodner E, Palgi Y (2020) COVID-19 health worries and anxiety symptoms among older adults: the moderating role of ageism. Int Psychogeriatr. https:// doi.org/10.1017/S1041610220001258

Bratt C, Abrams D, Swift HJ, Vauclair CM, Marques S (2018) Perceived age discrimination across age in Europe: from an ageing society to a society for all ages. Dev Psychol 54:167-180. https:// doi.org/10.1037/dev0000398

Bruine de Bruin W, Bennett D (2020) Relationships between initial COVID-19 risk perceptions and protective health behaviors: a national survey. Am J Prev Med 59:157-167. https://doi.org/10. 1016/j.amepre.2020.05.001

Cesari M, Proietti M (2020) COVID-19 in Italy: ageism and decision making in a pandemic. J Am Med Dir Assoc 21:576-577. https:// doi.org/10.1016/j.jamda.2020.03.025

Chang ES, Kannoth S, Levy S, Wang SY, Lee JE, Levy BR (2020) Global reach of ageism on older persons' health: a systematic review. PLoS ONE 15:e220857. https://doi.org/10.1371/journ al.pone. 0220857

Chasteen AL, Cary LA, Iankilevitch M (2017) Age stereotyping and discrimination. In: Pachana NA (ed) Encyclopedia of geropsychology. Springer, Singapore, pp 1-10. https://doi.org/10.1007/ 978-981-287-082-7_4

Cohn-Schwartz E, Ayalon L (2020) Societal views of older adults as vulnerable and a burden to society during the COVID-19 outbreak: results from an Israeli nationally representative sample. J Gerontol B Psychol Sci Soc Sci. https://doi.org/10.1093/geronb/ gbaa 150

Ehni HJ, Wahl HW (2020) Six propositions against ageism in the COVID-19 pandemic. J Aging Soc Policy 32:515-525. https:// doi.org/10.1080/08959420.2020.1770032

Garstka TA, Schmitt MT, Branscombe NR, Hummert ML (2004) How young and older adults differ in their responses to perceived age discrimination. Psychol Aging 19:326-335. https://doi.org/10. 1037/0882-7974.19.2.326

Han J (2018) Chronic illnesses and depressive symptoms among older people: functional limitations as a mediator and self-perceptions of aging as a moderator. J Aging Health 30(8):1188-1204. https:// doi.org/10.1177/0898264317711609

Hayes A (2017) Introduction to mediation, moderation, and conditional process analysis: a regression-based approach, 2nd edn. The Guilford Press, New York

Kornadt AE, Kessler E-M, Wurm S, Bowen CE, Gabrian M, Klusmann V (2020) Views on ageing: a lifespan perspective. Eur J Ageing 17:387-401. https://doi.org/10.1007/s10433-019-00535-9

Kotter-Gruhn D, Kornadt AE, Stephan Y (2016) Looking beyond chronological age: current knowledge and future directions in the study of subjective age. Gerontology 62:86-93. https://doi.org/ $10.1159 / 000438671$

Krendl AC, Perry BL (2020) The impact of sheltering-in-place during the COVID-19 pandemic on older adults' social and mental 
well-being. J Gerontol B Psychol Sci Soc Sci. https://doi.org/10. 1093/geronb/gbaa110

Levy B (2009) Stereotype embodiment: a psychosocial approach to aging. Curr Dir Psychol Sci 18:332-336. https://doi.org/10.1111/j. 1467-8721.2009.01662.x

Levy BR, Leifheit-Limson E (2009) The stereotype-matching effect: greater influence on functioning when age stereotypes correspond to outcomes. Psychol Aging 24:230-233. https://doi.org/10.1037/ a0014563

Levy BR, Slade MD, Chang ES, Kannoth S, Wang SY (2020) Ageism amplifies cost and prevalence of health conditions. Gerontologist 60:174-181. https://doi.org/10.1093/geront/gny131

Lichtenstein B (2020) From "coffin dodger" to "boomer remover:" outbreaks of ageism in three countries with divergent approaches to coronavirus control. J Gerontol B Psychol Sci Soc Sci. https:// doi.org/10.1093/geronb/gbaa102

Losada-Baltar A, Jimenez-Gonzalo L, Gallego-Alberto L, PedrosoChaparro MDS, Fernandes-Pires J, Marquez-Gonzalez M (2020) We're staying at home association of self-perceptions of aging, personal and family resources and loneliness with psychological distress during the lock-down period of COVID-19. J Gerontol B Psychol Sci Soc Sci. https://doi.org/10.1093/geronb/gbaa048

Meisner BA (2020) Are you ok, boomer? Intensification of ageism and intergenerational tensions on social media amid COVID-19. Leis Sci. https://doi.org/10.1080/01490400.2020.1773983

Monahan C, Macdonald J, Lytle A, Apriceno M, Levy SR (2020) COVID-19 and ageism: how positive and negative responses impact older adults and society. Am Psychol. https://doi.org/10. 1037/amp0000699

Montero-Odasso M, Hogan DB, Lam R, Madden K, MacKnight C, Molnar F, Rockwood K (2020) Age alone is not adequate to determine healthcare resource allocation during the COVID-19 pandemic. Can Geriatr J 23:152-154. https://doi.org/10.5770/cgj. 23.452

Morrow-Howell N, Gonzales E (2020) Recovering from coronavirus disease 2019 (COVID-19): resisting ageism and recommitting to a productive aging perspective. Public Policy Aging Rep. https:// doi.org/10.1093/ppar/praa021

Onder G, Rezza G, Brusaferro S (2020) Case-fatality rate and characteristics of patients dying in relation to COVID-19 in Italy. JAMA 323:1775-1776. https://doi.org/10.1001/jama.2020.4683

Ryan EB, Meredith SD, MacLean MJ, Orange JB (1995) Changing the way we talk with elders: promoting health using the communication enhancement model. Int J Aging Hum Dev 41:89-107. https://doi.org/10.2190/FP05-FM8V-0Y9F-53FX

Segel-Karpas D, Palgi Y, Shrira A (2017) The reciprocal relationship between depression and physical morbidity: the role of subjective age. Health Psychol 36:848-851. https://doi.org/10.1037/hea00 00542
Shrira A, Palgi Y, Hoffman Y, Avidor S, Bodner E, Ben-Ezra M, Bensimon M (2018) Subjective age as a moderator in the reciprocal effects between posttraumatic stress disorder symptoms and selfrated physical functioning. Front Psychol 9:1746. https://doi.org/ 10.3389/fpsyg.2018.01746

Steverink N, Westerhof GJ, Bode C, Dittmann-Kohli F (2001) The personal experience of aging, individual resources, and subjective well-being. J Gerontol B Psychol Sci Soc Sci 56:364-373. https:// doi.org/10.1093/geronb/56.6.P364

van Tilburg TG, Steinmetz S, Stolte E, van der Roest H, de Vries DH (2020) Loneliness and mental health during the COVID-19 pandemic: a study among dutch older adults. J Gerontol B Psychol Sci Soc Sci. https://doi.org/10.1093/geronb/gbaa111

Vauclair CM, Marques S, Lima ML, Abrams D, Swift H, Bratt C (2015) Perceived age discrimination as a mediator of the association between income inequality and older people's self-rated health in the European region. J Gerontol B Psychol Sci Soc Sci 70:901-912

Vogt Yuan AS (2007) Perceived age discrimination and mental health. Soc Forces 86:291-311

Weiss D, Sassenberg K, Freund AM (2013) When feeling different pays off: How older adults can counteract negative age-related information. Psychol Aging 28:1140-1146. https://doi.org/10. 1037/a0033811

Westerhof GJ, Miche M, Brothers AF, Barrett AE, Diehl M, Montepare JM, Wahl H-W, Wurm S (2014) The influence of subjective aging on health and longevity: a meta-analysis of longitudinal data. Psychol Aging 29(4):793-802. https://doi.org/10.1037/a0038016

Whitley E, Popham F, Benzeval M (2016) Comparison of the RoweKahn model of successful aging with self-rated health and life satisfaction: the West of Scotland twenty-07 prospective cohort study. Gerontologist 56:1082-1092

Wurm S, Warner LM, Ziegelmann JP, Wolff JK, Schüz B (2013) How do negative self-perceptions of aging become a self-fulfilling prophecy? Psychol Aging 28:1088-1097. https://doi.org/10.1037/ a0032845

Wurm S, Diehl M, Kornadt AE, Westerhof GJ, Wahl H-W (2017) How do views on aging affect health outcomes in adulthood and late life? Explanations for an established connection. Dev Rev 46:27-43. https://doi.org/10.1016/j.dr.2017.08.002

Xiang X, Lu X, Halavanau A, Xue J, Sun Y, Lai PHL, Wu Z (2020) Modern senicide in the face of a pandemic: An examination of public discourse and sentiment about older adults and COVID-19 using machine learning. J Gerontol B Psychol Sci Soc Sci. https:// doi.org/10.1093/geronb/gbaa128

Publisher's Note Springer Nature remains neutral with regard to jurisdictional claims in published maps and institutional affiliations. 